\title{
A CHARACTERIZATION OF EXCHANGE RINGS
}

\author{
G. S. MONK ${ }^{1}$
}

\begin{abstract}
A necessary and sufficient condition on the endomorphism ring of a module for the module to have the finite exchange property is given. This condition is shown to be strictly weaker than a sufficient condition given by Warfield. The class of rings having these properties is equationally definable and is a natural generalization of the class of regular rings. Finally, it is observed that in the commutative case the category of such rings is equivalent with the category of ringed spaces $(X, \mathscr{R})$ with $X$ a Boolean space and $\mathscr{R}$ a sheaf of commutative (not necessarily Noetherian) local rings.
\end{abstract}

In all that follows we will consider modules and morphisms as being over a ring $S$. Given two direct decompositions $K=M \oplus X=\left(\oplus \sum\left\{A_{i} \mid i \in I\right\}\right.$ of a module, we say that they can be exchanged at $M$ if there are submodules $A_{i}^{\prime} \subseteq A_{i}(i \in I)$ such that $K=M \oplus\left(\oplus \sum\left\{A_{i}^{\prime} \mid i \in I\right\}\right)$. A module $M$ is said to have the $n$-exchange property if any pair of decompositions $K=M^{\prime} \oplus X=\oplus \sum\left\{A_{i} \mid i \in I\right\}$ with $M^{\prime} \cong M$ and card $(I) \leqq n$ can be exchanged at $M^{\prime}$. The module $M$ has the (finite) exchange property if it has the $n$-exchange property for all (finite) cardinals $n$. In a fundamental paper [1], Crawley and Jónsson define the exchange properties and use them to prove theorems on isomorphic refinements of direct decompositions of modules. Warfield has shown [3] that an indecomposable module has the exchange property if and only if its endomorphism ring is local. In an attempt to generalize this theorem he has shown recently [4] that if $R$ is $\operatorname{End}_{S}(M)$ and $J$ is its Jacobson radical, then $M$ has the finite exchange property if every principal left ideal of $R / J$ is of the form $R e+J$ where $e$ is an idempotent element in $R$. In the present paper we will give another condition on $R$ which is, in fact, necessary and sufficient for $M$ to have the finite exchange property and furthermore is strictly weaker than Warfield's.

We will frequently use the fact that a direct decomposition $K=A B B$ of a module is naturally related to an idempotent endomorphism $\pi$ of $K$ which leaves the elements of $A$ fixed and has $B$ as its kernel. This will be

Presented to the Society, June 28, 1971; received by the editors January 24, 1972.

AMS 1970 subject classifications. Primary 16A30, 16A32.

Key words and phrases. Exchange property, isomorphic refinement of decompositions, equational classes of rings, representation of commutative rings.

${ }^{1}$ This research was supported by National Science Foundation grant GP-28946. 
referred to as the projection on $A$ along $B$ and denotc 1 by $K=A \oplus B$; $\pi: K \rightarrow A$. We will routinely abuse notation by beginning $v$ ith a morphism $f: A_{1} \rightarrow A_{2}$ and submodules $B_{i} \subseteq A_{i}(i=1,2)$ such that $f\left(B_{1}\right) \subseteq B_{2}$ and writing $f: B_{1} \rightarrow B_{2}$ instead of $\left(f \mid B_{1}\right): B_{1} \rightarrow B_{2}$.

Our point of view is that if $K=M \oplus X=A \oplus B$ and $\pi$ is $t^{2}$ e projection on $A$ along $B$, then exchange at $M$ can 'e characterized in terms of the action of $\pi$ on $M$, which is, in turn, dircribable as a firs $\ddagger$ order sentence about $R=\operatorname{End}_{S}(M)$.

Definition. Given a module $K$ with submodule $M$, the morphism $\alpha$ of $M$ into $K$ is divided on $M$ if $M=\Lambda_{L_{1}}^{*} \oplus M_{2}$ anc $K=K_{1} \oplus K_{2}$ such that (i) $\alpha: M_{1} \cong K_{1}$, (ii) $\alpha\left(M_{2}\right) \subseteq K_{2}$, (iii) $1-\alpha: M_{2} \gg K$ s slits.

It is not difficult to show that the encomorphisi ? ring $R$ of a module $M$ is regular if and only if given $\alpha \in R, M=M_{1} \oplus \operatorname{ker}(\alpha)$ such that $\alpha: M_{1}>\rightarrow M$ splits. This condition clearly implies that $\alpha: M \rightarrow M$ is divided on $M$.

Lemma 1. Given a module $K$ with submo tule $M$, the morphism $\alpha: M \rightarrow K$ is divided on $M$ if and only if there are morp hisms $\gamma$ ('nd $\sigma$ in $\operatorname{Hom}_{S}(K, M)$ such that $\gamma \alpha \gamma^{\prime}=\gamma$ on $K$, and $\sigma\left(1_{M}-\alpha\right)\left(1_{M}-\gamma \alpha\right)=1_{1 i}-\gamma \alpha$ on $M$.

Proof. Given $\gamma, \gamma$ and $\sigma$ with these properties, ojserve that $\alpha \gamma$ and $\gamma \alpha$ are idempotent endomorphisms of $K$ and $M$ respectively and that $\left(1_{M}-\alpha\right):\left(1_{M}-\gamma \alpha\right)(M) \curvearrowright K$ splits. But then $\alpha$ divides on $M$ because we have the decompositions

$$
M=M_{1} M_{2}: \quad \gamma \alpha(M)=M_{1}, \quad K=K_{1} \oplus K_{2} ; \quad \alpha \gamma(K)=K_{1} .
$$

Conversely, if $M=M_{1} \oplus M_{2}$ and $K=K_{1} \oplus K_{2}$ such that $\alpha: M_{1} \cong K_{1}$, $\alpha\left(M_{2}\right) \subseteq K_{2}$ and $\left(1_{M}-\alpha\right): M_{2}>K$ splits, then, defining $\gamma$ on $K_{1}$ as $\left(\alpha \mid M_{1}\right)^{-1}$ and on $K_{2}$ as 0 , we have that $\alpha \gamma$ is the projection on $K_{1}$ along $K_{2}$, while $\gamma \alpha$ is the projection on $M_{1}$ along $M_{2}$. But then the equations follow easily.

Lemma 2. The decompositions $K=M \oplus X, K=A \oplus \beta, \pi(K)=A$ can be exchanged at $M$ if and only if $\pi$ is divided on $M$.

Proof. If $K=M \oplus A_{1} \oplus B_{1}$ with $A_{1} \subseteq A$ and $B_{1} \subseteq B$, then, letting

$$
\begin{aligned}
M_{1} & =M \cap\left(A+B_{1}\right), & M_{2} & =M \cap\left(A_{1}+B\right), \\
A_{2} & =A \cap\left(M+B_{1}\right), & K_{2} & =A_{1}+B,
\end{aligned}
$$

it can be shown easily that $M=M_{1} \oplus M_{2}, K=A_{2} \oplus K_{2}, \pi: M_{1} \cong A_{2}$, and $\pi\left(M_{2}\right) \subseteq K_{2}$. Since $\left(1_{,}-\pi\right)$ is monic on $M_{2}$ and $(1-\pi)\left(M_{2}\right)=B \cap\left(M+A_{1}\right)$, which is a complement of $A+B_{1}$ in $K$, we conclude that $\pi$ is divided on $M$.

For the converse suppose that there are endomorphisms $\gamma$ and $\sigma$ such that $\gamma \pi \gamma=\gamma$ on $K$ and

$$
\sigma\left(1_{. Y}-\pi\right)\left(1_{. Y}-\gamma \pi\right)=1_{. Y}-\gamma \pi \text { on } M .
$$


Then, noting that if $\rho$ is the projection on $M$ along $X, \gamma=\rho \gamma,\left(1_{M}-\gamma \pi\right) \rho=$ $\rho\left(1_{M}-\gamma \pi\right) \rho=\left(1_{K}-\gamma \pi\right) \rho$ and $\left(1_{M}-\pi\right) \rho=\left(1_{K}-\pi\right) \rho$, multiplying (1) on the right by $\rho$ and on the left by $\left(1_{K}-\gamma \pi\right)$, we obtain

$$
\left(1_{K}-\gamma \pi\right) \sigma\left(1_{K}-\pi\right)\left(1_{K}-\gamma \pi\right) \rho=\left(1_{K}-\gamma \pi\right) \rho \quad \text { on } K .
$$

Now if we let $\theta_{1}$ be the idempotent $\gamma \pi$ and $\theta_{2}=\left(1_{K}-\theta_{1}\right) \sigma\left(1_{K}-\pi\right)\left(1_{K}-\theta_{1}\right)$, we infer from (2) that $\theta_{2}$ is an idempotent such that

and

$$
\theta_{1} \rho+\theta_{2} \rho=\rho, \quad \theta_{1} \theta_{2}=\theta_{2} \theta_{1}=0
$$

$$
\theta_{2} \pi\left(1_{K}-\theta_{1}\right)=\left(1_{K}-\theta_{1}\right) \sigma\left(1_{K}-\pi\right)\left(\pi-\theta_{1}\right)\left(1_{K}-\theta_{1}\right)=0 .
$$

Thus, if we let $\theta_{i}(K)=M_{i}, \operatorname{ker} \theta_{i}=L_{i}(i=1,2)$, we obtain $M=M_{1}+M_{2}$ :

$$
\begin{array}{ll}
K=M_{1} \oplus L_{1}, & B, M_{2} \subseteq L_{1}, \\
K=M_{2} \oplus L_{2}, & M_{1},\left(A \cap L_{1}\right) \subseteq L_{2},
\end{array}
$$

so that

$$
\begin{aligned}
L_{1} & =(A \oplus B) \cap L_{1}=\left(A \cap L_{1}\right) \oplus B, \\
L_{1} \cap L_{2} & =\left(A \cap L_{1}\right) \oplus\left(B \cap L_{2}\right), \\
L_{2} & =\left(M_{1} \oplus L_{1}\right) \cap L_{2}=M_{1} \oplus\left(L_{1} \cap L_{2}\right) \\
& =M_{1} \oplus\left(A \cap L_{1}\right) \oplus\left(B \cap L_{2}\right)
\end{aligned}
$$

and

$$
\begin{aligned}
K & =M_{2} \oplus M_{1} \oplus\left(A \cap L_{1}\right) \oplus\left(B \cap L_{2}\right) \\
& =M \oplus\left(A \cap L_{1}\right) \oplus\left(B \cap L_{2}\right),
\end{aligned}
$$

which is the desired result.

THEOREM 1. The module $M$ has the finite exchange property if and only if the ring $R=\operatorname{End}_{S}(M)$ has the property

(E) Given $\alpha \in R$, there exist $\gamma$ and $\sigma$ in $R$ such that $\gamma \alpha \gamma=\gamma$ and $\sigma(1-\alpha)(1-\gamma \alpha)=1-\gamma \alpha$.

Proof. We first recall that, according to Crawley and Jónsson [1, Lemma 3.11], the finite exchange property for a module $M$ is equivalent to the 2-exchange property. If the condition (E) holds for $R$ and

$$
K=M \oplus X=A \oplus B, \quad \rho(K)=M, \quad \pi(K)=A,
$$

then $\rho \pi \rho$, viewed as an endomorphism of $M$, is divided on $M$. It is then easily seen that $\pi$ as a morphism of $M$ into $K$ is divided on $M$, whence the decompositions (3) can be exchanged at $M$ and $M$ has the finite exchange property. 
To prove the converse we fix $\alpha \in R$ and define $K=M \times M$,

$$
M \underset{\tau_{1}}{\stackrel{\varepsilon_{1}}{\rightleftarrows}} K \underset{\tau_{2}}{\stackrel{\varepsilon_{2}}{\rightleftarrows}} M
$$

$\sigma(m)=(\alpha(m),(1 \cdots \alpha)(m))$ and $\Delta(m)=(m,-m)$. Then 'et $M_{\sigma}, \mathcal{M}_{\Delta}, A$ and $B$ be the images of $M$ in $K$ under $\sigma, \Delta, \varepsilon_{1}$ and $\varepsilon_{2}$ respcctively. It is clear that $\sigma$ is an isomorphism of $M$ onto $M_{\sigma}$ and that $K=I_{\sigma} r_{\sigma} \oplus M_{\Delta}=A \oplus B$ with $\varepsilon_{1} \tau_{1}$ the projection on $A$ along $B$. Since $M$ has the 2 exchange property, these decompositions can be exchanged at $M_{\sigma}$ and $\varepsilon_{1} \tau_{1}$ is divided on $M_{\sigma}$ with $M_{\sigma}=M_{\sigma}^{(1)}\left(M_{\sigma}^{(2)}\right.$ and $K=A_{2} \oplus K_{2}$ the related decompositions. Inasmuch as $A_{2} \subseteq A$, we infer from the second decomposition that $A=$ $A_{2} \oplus\left(A \cap K_{2}\right)$. Now the isomorphisms $\sigma: M \cong M_{\sigma}$ and $\varepsilon_{1}: M \cong A$ give decompositions $\quad M=M_{1} \oplus M_{2}=M_{3} \oplus M_{4}$ where $\sigma\left(M_{i}\right)=M_{\sigma}^{(i)} \quad(i=1,2)$, $\varepsilon_{1}\left(M_{3}\right)=A_{2}$ and $\varepsilon_{1}\left(M_{4}\right)=A \cap K_{2}$. It is then easy to show that $\tau_{1}\left(\varepsilon_{1} \tau_{1}\right) \sigma$ : $M_{1} \cong M_{3}, \tau_{1}\left(\varepsilon_{1} \tau_{1}\right) \sigma\left(M_{2}\right) \subseteq M_{4}$ and that $\tau_{2}\left(1-\varepsilon_{1} \tau_{1}\right) \sigma: I I_{2}>\rightarrow M$ splits. But since $\alpha=\tau_{1}\left(\varepsilon_{1} \tau_{1}\right) \sigma$ and $(1-\alpha)=\tau_{2}\left(1-\varepsilon_{1} \tau_{1}\right) \sigma$, we conclude that $\alpha$ is divided on $M$ which gives the desired result.

Warfield defines a ring $R$ to be an exchange ring if ${ }_{R} R$ has the exchange property (as an $R$-module), but then goes on to show that such rings are exactly the rings of endomorphisms of modules with the finite exchange property [4, Theorem 2]. Thus we have characterized exchange rings in Theorem 1. As was mentioned in the introduction, Warfield also proves [4, Theorem 3] that if $R$ is a ring such that every principal left ideal of $R / J$ is of the form $R e+J$ where $e=e^{2}$ and $J$ is the Jacobson radical of $R$, then $R$ is an exchange ring. Our next theorem shows that this does not characterize exchange rings.

THEORFM 2. There is a commutative exchange ring which is Jacobson semisimple but not regular.

Proor. Let $Z_{\text {, }}$ be the $p$-adic integers, $F$ any field having $\hat{Z}_{p}$ as a subring and $A$ the ring product of $\aleph_{0}$ copies of $F$. Viewing $A$ as sequences of elements from $F$, let $R$ be the subring of $A$ generated by the finitely nonzero sequences of $A$ (called $A_{0}$ ) and multiples from $\hat{Z}_{p}$ of $(1,1,1, \cdots$ ). It is clear that if, for $a \in A$, we denote by $\pi_{i}(a)$ the $i$ th coordinate of $a$, then $\pi_{i}: R \rightarrow F$ so that $\operatorname{ker} \pi_{i}$ is a maximal ideal in $S$. Inasmuch as $\bigcap\left\{\operatorname{ker} \pi_{i} \mid i=1,2,3, \cdots\right\}=(0), R$ is a Jacobson semisimple ring. But $R$ cannot be regular because the factor rings of such a ring would be regular and $\hat{Z}_{p}$, which is isomorphic to $R /\left(A_{0}\right)$, is not regular. To see that $R$ is an exchange ring, begin with a typical element $a=\left(a_{1}, a_{2}, \cdots, a_{m}, r, r, \cdots\right)$ in $R$ where $a_{i} \in t, 1 \leqq i \leqq m$, and $r \in \hat{Z}_{p}$. For each $i=1,2, \cdots, m$ such that $a_{i} \neq 0$, let $c_{i}=a_{i}{ }^{1}$ and $d_{i}=0$. For $i=1,2, \cdots, m$ such that $a_{i}=0$ let $c_{i}=d_{i}=0$. Then for $1 \leqq i \leqq m, c_{i} a_{i} c_{i}=c_{i}$ and $d_{i}\left(1-a_{i}\right)\left(1-a_{i} c_{i}\right)=1-a_{i} c_{i}$. 
If $r \notin p \hat{Z}_{p}$, let $s=r^{-1}$ in $\hat{Z}_{p}$ and $t=0$. If $r \in p \hat{Z}_{p}$, let $s=0$ and $t=$ $(1-r)^{-1}$. In either case $s r s=s$ and $t(1-r)(1-r s)=1-r s$, and if we let $c=$ $\left(c_{1}, \cdots, c_{m}, s, s, \cdots\right)$ and $d=\left(d_{1}, \cdots, d_{m}, t, t, \cdots\right)$ the desired equations hold for $a, c$ and $d$.

Constructions of the sort used in the preceding proof can be viewed as a special case of the formation of the ring of sections of a sheaf of rings over a Boolean space. In view of the fact that Pierce [2] uses this technique to such advantage in his study of commutative regular rings, it seems natural to study commutative exchange rings in connection with ringed spaces, although this is far removed from the original source of exchange rings. However, recalling Warfield's characterization of an exchange ring with no nontrivial idempotents as a local ring (in the sense of having a unique maximal ideal) [2, Proposition 1], we have the following pleasant result.

THEOREM 3. There is an equivalence between the category of commutative exchange rings and the category of ringed spaces $(X, \mathscr{R})$ with $X$ a Boolean space and $\mathscr{R}$ a sheaf of commutative local rings.

Proof. First observe that if $(X, \mathscr{R})$ is a ringed space with $X$ a Boolean space and $\mathscr{R}$ a sheaf of commutative local rings, then each of the stalks satisfies the property (E), so that, by Pierce's Theorem 3.4 [2], the ring of sections of $(X, \mathscr{R})$ does likewise. On the other hand, the stalks of the ringed space of a commutative exchange ring are commutative exchange rings (since the property (E) is preserved by epimorphisms) with no nontrivial idempotents and hence are local. Pierce's Theorem 10.1 then gives the equivalence of these categories.

\section{BIBLIOGRAPHY}

1. P. Crawley and B. Jónsson, Refinements for infinite direct decompositions of algebraic systems, Pacific J. Math. 14 (1964), 797-855. MR 30 \#49.

2. R. S. Pierce, Modules over commutative regular rings, Mem. Amer. Math. Soc. No. 70 (1967). MR 36 \#151.

3. R. B. Warfield, Jr., A Krull-Schmidt theorem for infinite sums of modules, Proc. Amer. Math. Soc. 22 (1969), 460-465. MR 39 \#4213.

4. - Exchange rings and decompositions of modules, Math. Ann. (to appear). 